\title{
АДАПТИВНАЯ ФИЛЬТРАЦИЯ ГОЛОГРАФИЧЕСКОГО ИЗОБРАЖЕНИЯ
}

Головинский Павел Абрамович доктор физ.-мат. наук, профессор кафедры инноватики и строительной физики имени профессора И. С. Суровцева

Тарасова Анна Сергеевна

Магистрант кафедры инноватики и строительной физики имени профессора И. С. Суровцева «Воронежский государственный технический университет» (ФГБОУ ВО «ВГТУ», ВГТУ)

\begin{abstract}
Аннотация: в работе рассмотрено восстановление голографического изображения с помощью дифракционного интеграла Френеля-Кирхгофа при наличии точечных шумов в рамках цифровой голографии Фурье; показано применение медианного фильтра для очистки поля изображения для приосевой голографии, а так же применение обучаемого комплекснозначного линейного фильтра для внеосевой голографии.

Ключевые слова: цифровая голография, искусственные нейронные сети, восстановление данных, комплексные нейронные сети, голография Фурье, автокодировщик.
\end{abstract}

\section{ADAPTIVE FILTERING OF THE HOLOGRAPHIC IMAGE}

\section{Golovinski Pavel Abramovich Tarasova Anna Sergeevna}

\begin{abstract}
: the paper considers the restoration of a holographic image using the Fresnel-Kirchhoff diffraction integral in the presence of point noise in the framework of Fourier digital holography; shows the use of a median filter to clear the image field for paraxial holography, as well as the use of a trainable complex-valued linear filter for off-axis holography.
\end{abstract}

Keywords: digital holography, artificial neural networks, data recovery, complex neural networks, Fourier holography, autoencoder. 


\section{1. Введение}

Как было показано Д. Габором [1, с. 777-778.], волновое поле, характеризуемое амплитудами и фазами в различных точках пространства, может быть записано с помощью распределения интенсивностей интерферирующих полей опорной и объектной волн, а затем воспроизведено путем подсветки полученной голограммы. Фиксация изображения в отдельных зернах фотоэмульсии навела на мысль о дискретном представлении в голографии, что привело к созданию и развитию компьютерной голографии [2, c. 27; 3, c 1-7].

Альтернативой цифровой голографии является подход, в котором поведение отдельных элементов голограммы при записи и воспроизведении описывается соответствующим нейроном с комплексными входами и комплексным выходом. Построенная на таких нейронах память способна воспроизводить исходное поле, как отклик на однородный входной сигнал [4, с. 259-267; 5, с. 1637-1645]. Одной из актуальных современных задач виртуальной реальности является создание полноценного голографического кино и телевидения. В качестве возможной технологии производства кинофильмов продемонстрировано использование метаповерхностных голограмм, в которых плоская поверхность содержит ячейки из металлических или диэлектрических элементов размером меньше длины волны оптического излучения [6, с. 23761-23770]. Особый интерес представляет реализация голографических очков с помощью специальных голографических оптических модуляторов [7, с. 1563-1578]. Достижения в области метаповерхностей и жидких кристаллов позволили сконструировать цветную и зависящую от поляризации света голографическую систему [8, с. 119-124; 9, с. 35631-35645]. Такие системы могут применяться как с использованием линз, так и более компактно на основе дифракции Френеля.

Отмеченные тенденции делают актуальной задачу синтеза голографического изображения с улучшением его качества. Для коррекции обычного не голографического изображения используются различные цифровые фильтры [10, с. 1103], включая алгоритмы автокодировки [11, с. 899-907]. Поскольку в случае голографического изображения исходные данные являются скалярными полями, а считывающая и восстанавливаемая волны описываются комплексными величинами, то, следовательно, требуется определенная модификация известных алгоритмов цифровой обработки изображений. $\mathrm{B}$ данной работе предложены двухступенчатые линейные 
адаптивные фильтры, улучшающие сначала голограмму, а затем полученное восстановленное изображение.

\section{2. Основные уравнения цифровой голографии Фурье}

Конкретный вид цифровых голографических преобразований определяется приближениями, которые используются при описании дифракции. Приосевые голограммы (in-line holograms) формируются с помощью плоской опорной волны $\mathrm{R}=\exp (\mathrm{ikz}+\mathrm{i} \varphi)$. Если расстояние от области записи голограммы до источника опорной волны велико, то комплексная амплитуда объектного поля $O(\xi, \eta, L)$ в плоскости записи голограммы $\{\xi, \eta, L\}$ в дальней области дифракции на расстоянии L от объекта может быть записана через распределение объектного поля $\mathrm{O}(\mathrm{x}, \mathrm{y}, 0)$ вблизи объекта в плоскости $\{\mathrm{x}, \mathrm{y}, 0\}$ в виде дифракционной формулы Фраунгофера $[12$, c. 620$]$ :

$$
\mathrm{O}(\xi, \eta, \mathrm{L})=\mathrm{A} \iint_{-\infty}^{\infty} \mathrm{O}(\mathrm{x}, \mathrm{y}, 0) \exp [-\mathrm{iS}] \mathrm{dxdy}
$$

где $\mathrm{A}=\frac{1}{\mathrm{i} \lambda \mathrm{L}} \exp \left[\mathrm{ikL}+\mathrm{ik}\left(\xi^{2}+\eta^{2}\right) / 2 \mathrm{~L}\right], \quad \mathrm{S}=\mathrm{ik}(\mathrm{x} \xi+\mathrm{y \eta}) / \mathrm{L}$, волновое число $\mathrm{k}=2 \pi / \lambda, \lambda-$ длина волны излучения. Экспоненциальный множитель перед интегралом с параболическим распределением фазы по поперечным координатам описывает сферическую волну в параксиальном приближении, когда $\mathrm{k}\left(\xi^{2}+\eta^{2}\right) / 2 \ll 1$.

На рис.1 показана прямоугольная апертура и результат расчета дифракции Фраунгофера на этой апертуре с помощью пакета прикладных программ MATLAB.

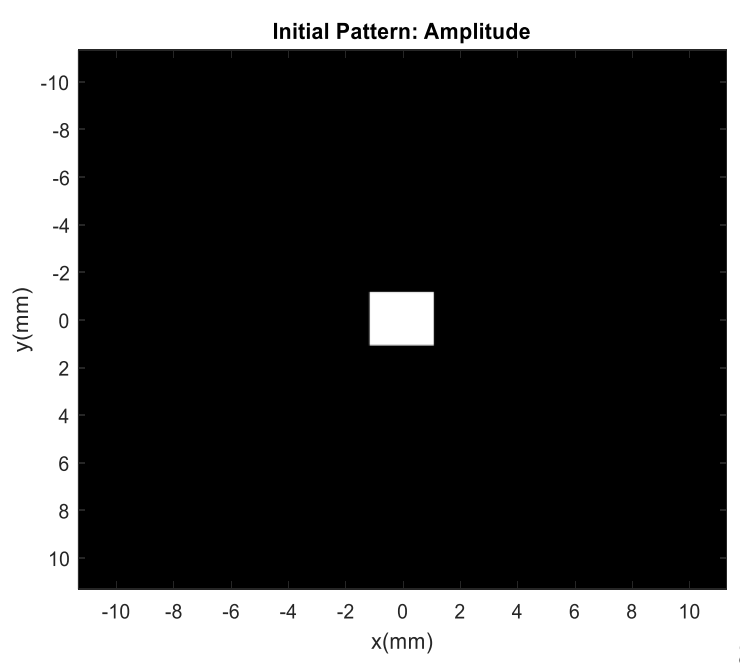

a)

b)

Рис.1. Дифракция Фраунгофера: а -прямоугольная апертур, b - результат дифракции. 
При записи голограммы регистрируется интенсивность $\mathrm{I}(\xi, \eta)$, возникающая в результате интерференции объектной волны (1) и опорной волны R:

$$
\mathrm{I}(\xi, \eta)=|\mathrm{O}(\xi, \eta, \mathrm{L})+\mathrm{R}|^{2}
$$

Перед реконструкцией голограммы необходимо вычесть из нее фон, соответствующий записи опорной волны в отсутствие объектной волны, так что

$$
\tilde{\mathrm{I}}(\xi, \eta)=\mathrm{I}(\xi, \eta)-1
$$

при $\mathrm{R}=1$. Если интенсивность объектной волны (1) намного меньше интенсивности опорной волны, то вклад в интенсивность голограммы квадратичного по $\mathrm{O}(\xi, \eta, L)$ слагаемого можно пренебречь, и для плоской опорной волны:

$$
\tilde{\mathrm{I}}(\xi, \eta) \approx 0(\xi, \eta, L)+0^{*}(\xi, \eta, L)
$$

Если использовать распределение (4) как модулирующий амплитуду падающей плоской волны транспарант, то результат дифракции, фиксируемый детектором в точке наблюдения $\{\mathrm{x}, \mathrm{y}, 0\}$ может быть найден повторным применением дифракционной формулы (1) с заменой $\mathrm{O}(\mathrm{x}, \mathrm{y}, 0) \rightarrow \tilde{\mathrm{I}}(\mathrm{x}, \mathrm{y}), \mathrm{k} \rightarrow$ $-\mathrm{k}$ и интегрированием в плоскости голограммы. Если опустить постоянные множители, включающие общую фазу, то результат двойного применения формулы Фраунгофера сводится к выражению

$$
\begin{gathered}
\mathrm{U}\left(\mathrm{x}^{\prime}, \mathrm{y}^{\prime}\right)=\frac{1}{4 \pi^{2}} \iint\left[\iint \mathrm{O}(\mathrm{x}, \mathrm{y}, 0) \mathrm{e}^{-\mathrm{iS}(\mathrm{x}, \mathrm{y})} \mathrm{dxdy}+\iint \mathrm{O}^{*}(\mathrm{x}, \mathrm{y}, 0) \mathrm{e}^{\mathrm{i} S(\mathrm{x}, \mathrm{y})} \mathrm{dxdy}\right] \times \\
\times \mathrm{e}^{\mathrm{i} \tilde{\mathrm{S}}(\xi, \eta)} \mathrm{d} \xi \mathrm{d} \eta,
\end{gathered}
$$

где $\widetilde{S}(\xi, \eta)=i k\left(x^{\prime} \xi+y^{\prime} \eta\right) / L$.

Для формально бесконечной голограммы, выполняя интегрирование для прямого и обратного двойного преобразования Фурье, получим выражение [13]:

$$
\mathrm{U}\left(\mathrm{x}^{\prime}, \mathrm{y}^{\prime}\right)=\mathrm{O}\left(\mathrm{x}^{\prime}, \mathrm{y}^{\prime}, 0\right)+\mathrm{O}^{*}\left(-\mathrm{x}^{\prime},-\mathrm{y}^{\prime}, 0\right)
$$




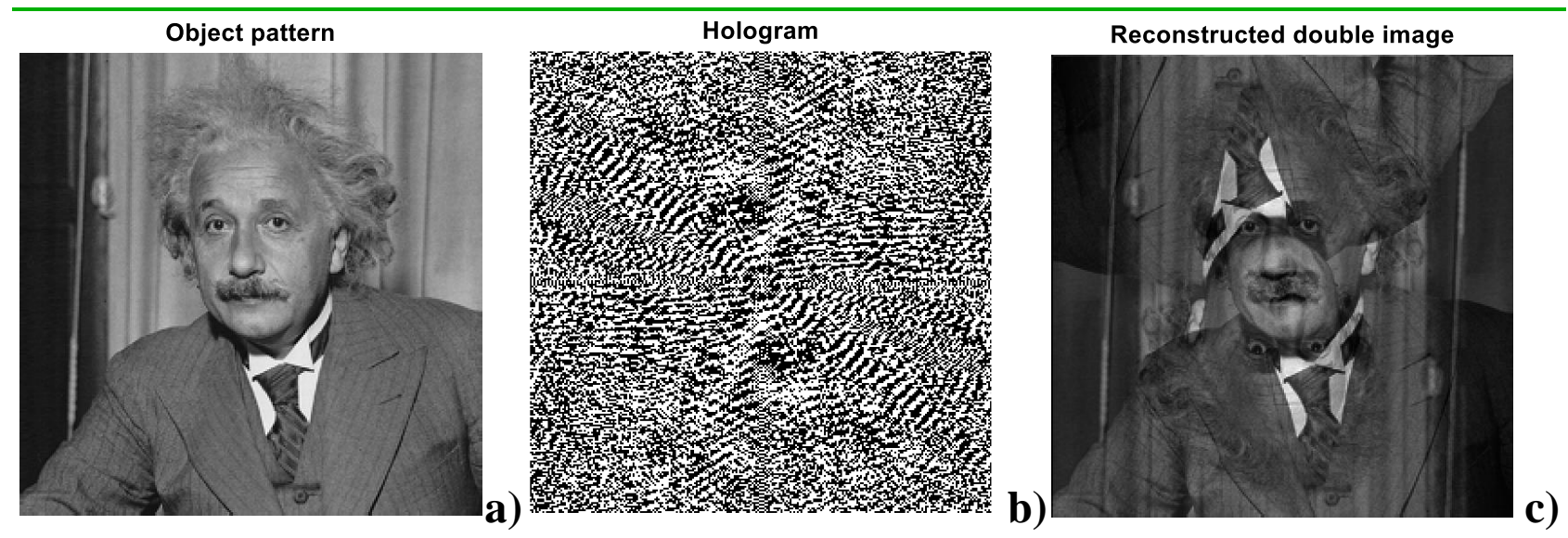

Рис. 2. Исходное изображение - a, голограмма Фурье - b, восстановленное изображение с наложением зеркального перевернутого двойника -c .

Формула (6) показывает, что действительное и мнимое изображения расположены симметрично относительно оптической оси и их фазы взаимно противоположны. Тем самым, наряду с основным возникает и второе размытое паразитное изображение, связанное со вторым слагаемым в уравнении (6), которое проявляется в виде помехи для распределения интенсивности восстановленного изображения (рис. 2).

Для устранения нежелательного двойственного изображения был предложен целый ряд решений [13 - 14; 15, с. 2339; 16, с. 572-578; 17, с. 1324 1328], мы воспользуемся идеей использовать симметрию восстановленного действительно изображения - Будем строить изначально изображение, разместив в четырех квадрантах начальное распределение поля

$$
\left(\begin{array}{ll}
\mathrm{Q}_{11} & \mathrm{Q}_{12} \\
\mathrm{Q}_{21} & \mathrm{Q}_{22}
\end{array}\right)=\left(\begin{array}{cc}
0 & \mathrm{O}(\mathrm{x}, \mathrm{y}, 0) \\
\mathrm{O}(-\mathrm{x},-\mathrm{y}, 0) & 0
\end{array}\right) .
$$

Найдем суперпозицию результата применения формулы (5) к $Q_{12}$ и $Q_{21}$. Тогда в результате восстановления по голограмме исходного изображения мы получим:

$$
\begin{gathered}
\mathrm{U}\left(\mathrm{x}^{\prime}, \mathrm{y}^{\prime}\right)=\mathrm{O}\left(\mathrm{x}^{\prime}, \mathrm{y}^{\prime}, 0\right)+\mathrm{O}^{*}\left(-\mathrm{x}^{\prime},-\mathrm{y}^{\prime}, 0\right)+\mathrm{O}\left(-\mathrm{x}^{\prime},-\mathrm{y}^{\prime}, 0\right)+\mathrm{O}^{*}\left(\mathrm{x}^{\prime}, \mathrm{y}^{\prime}, 0\right)= \\
=2 \operatorname{ReO}\left(\mathrm{x}^{\prime}, \mathrm{y}^{\prime}, 0\right)+2 \operatorname{ReO}\left(-\mathrm{x}^{\prime},-\mathrm{y}^{\prime}, 0\right) .
\end{gathered}
$$



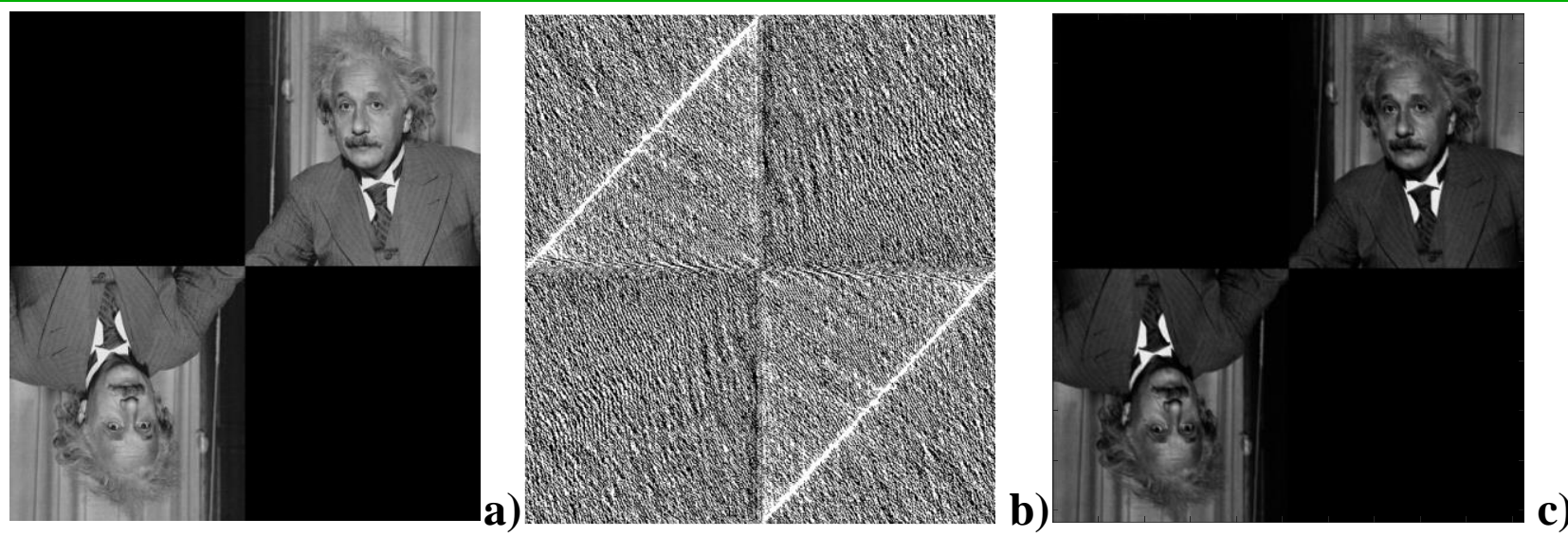

Рис. 3. Исходное изображение, размещенное в первом и третьем квадранте - a; голограмма, полученная с учетом маски - b; результат реконструкции исходного изображения - с.

Таким разделение поля (7) позволяет оптически или численно реконструировать действительную часть изображения без двойникования. Разделяя исходное изображение на действительную и мнимую части, можно записать их и восстановить с помощью масок по отдельности, исключая ложное изображение. При этом мнимую часть изображения можно обработать одновременно с действительной его частью, размещая исходные данные и их зеркало в свободной диагональной части матицы (рис. 3).

Рассмотрим также другой прием, позволяющий избавится от дублирующего изображения, для расчётов используем пакет прикладных программ MATLAB. Модулируем исходное изображение с некоторой частотой по координате

$$
\widetilde{\mathrm{O}}(\mathrm{x}, \mathrm{y}, 0)=\mathrm{O}(\mathrm{x}, \mathrm{y}, 0) \exp (\mathrm{i} \omega \mathrm{x}),
$$

при восстановлении используем фазовую функцию:

$$
\widetilde{\mathrm{S}}_{\omega}(\xi, \eta)=\frac{\mathrm{ik}\left(\mathrm{x}^{\prime} \xi+\mathrm{y}^{\prime} \eta\right)}{\mathrm{L}}-\mathrm{i} \omega \xi=\frac{\mathrm{ik}\left(\mathrm{x}^{\prime} \xi-\mathrm{d} \xi+\mathrm{y}^{\prime} \eta\right)}{\mathrm{L}}
$$

где $\mathrm{d}=\omega \mathrm{L} / \mathrm{k}$. В результате восстановленное изображение имеет вид

$$
\mathrm{U}\left(\mathrm{x}^{\prime}, \mathrm{y}^{\prime}\right)=\mathrm{O}\left(\mathrm{x}^{\prime}, \mathrm{y}^{\prime}, 0\right)+\mathrm{O}\left(-\mathrm{x}^{\prime}+\mathrm{d},-\mathrm{y}^{\prime}, 0\right)^{*},
$$

так что подходящим выбором смещения $\mathrm{d}$ мнимое и действительное изображения можно пространственно разделить. Здесь мы имеем компьютерный аналог вне осевого голографического метода Лейта-Упатниекса [17, с. 521-538] с наклонным падением волны. 

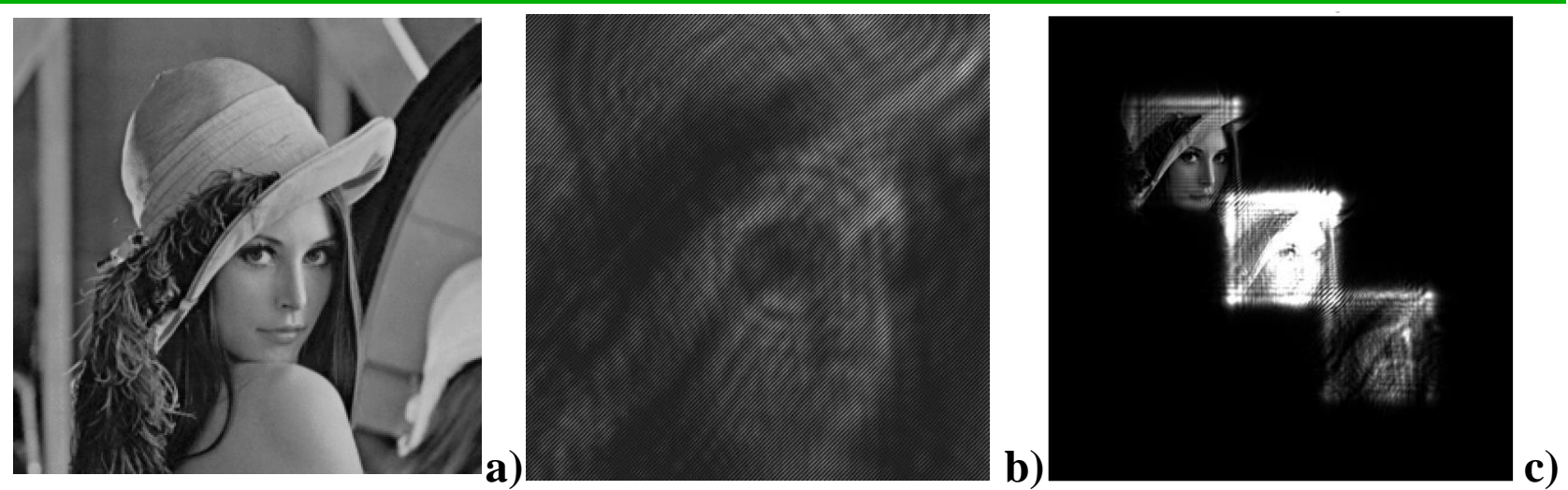

Рис. 4. Исходное изображение - a ; голограмма - b; результат реконструкции исходного изображения - с.

На рис. 4 показаны этапы записи и реконструкции голографического изображения при использовании вне осевого метода. Отметим, что качество восстановленного изображения существенно уступает результату, достигаемому при использовании квадрантной маски.

\section{3. Адаптивная фильтрация}

Для построения качественного голографического изображения наряду с традиционными методами голографии используются методы машинного обучения, заменяющие преобразования Фурье обученными с помощью алгоритмов глубокого обучения многослойными нейронными сетями [19 - 21; 22, с. 26636-26650]. Этот подход использует возможности преимущественно сверточных нейронных сетей для построения необходимых отображений на основе обучения без апелляции к оптическим явлениям, которые отвечают за моделируемый процесс. Результаты такого подхода демонстрируют не только высокое быстродействие, но и высокую устойчивость к шумам [23 - 24; 25, с. 440-444]. Однако к его недостаткам можно отнести то, что он не использует особенности физического процесса голографирования, и полученные результаты не имеют явной смысловой интерпретации и не дают качественного объяснения наблюдаемым эффектам.

Пример восстановления и очистки голограммы с помощью искусственной нейронной сети свёрточного автокодировщика, реализованного нами стандартными средствами библиотеки РуTorch на языке программирования Python 3.8 приведен на рис. 5-6. Для очистки голограммы мы использовали комбинацию из свёрточной нейросети и гауссова низкочастотного фильтра подавления высоких частот, который позволяет снизить влияние повреждения отдельных пикселей на качество изображения в виде 


$$
H(\xi, \eta)=\exp \left(-\frac{\xi^{2}+\eta^{2}}{2 D_{0}^{2}}\right)
$$

Частота среза $D_{0}$ подбирается из условия минимизации значения функции ошибок (13).

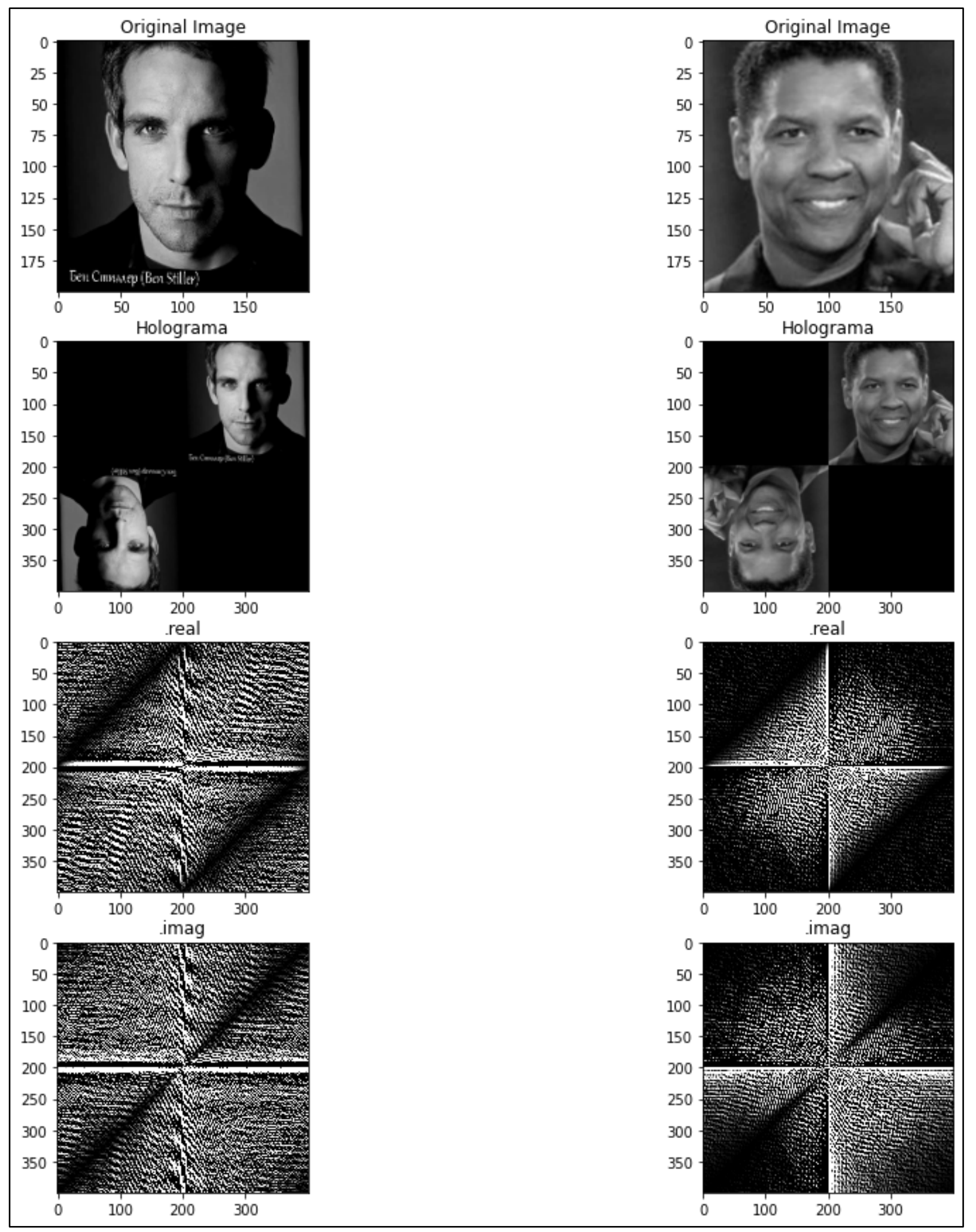

Рис. 5. Создание голографического набора данных в Python, для обучения искусственной нейронной сети 
A
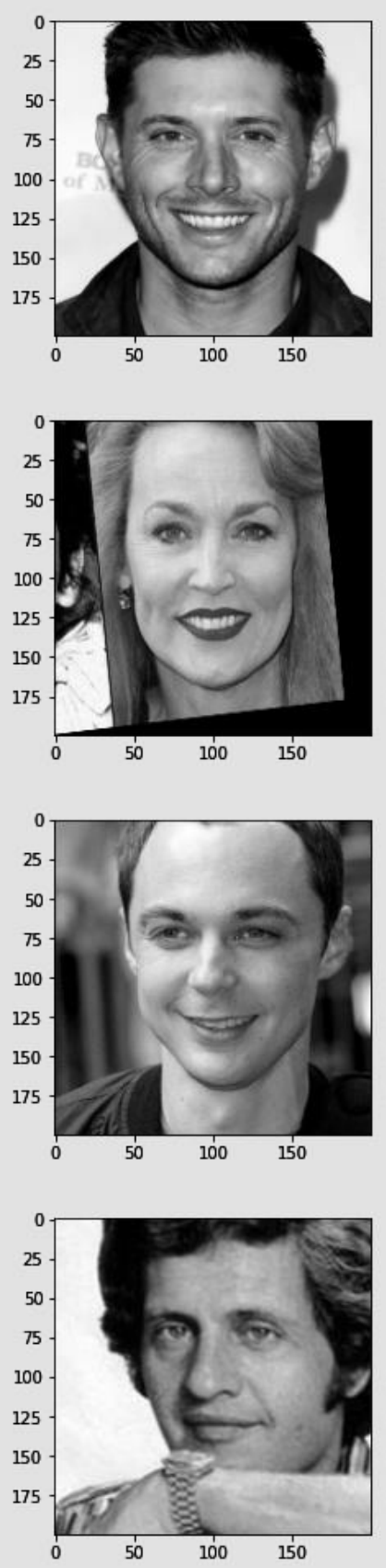

B
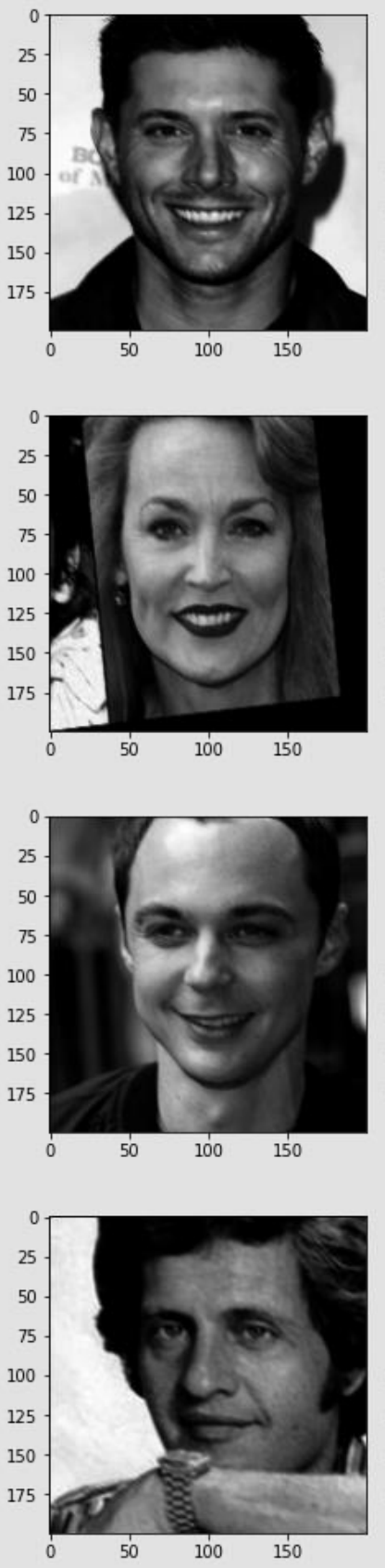

C
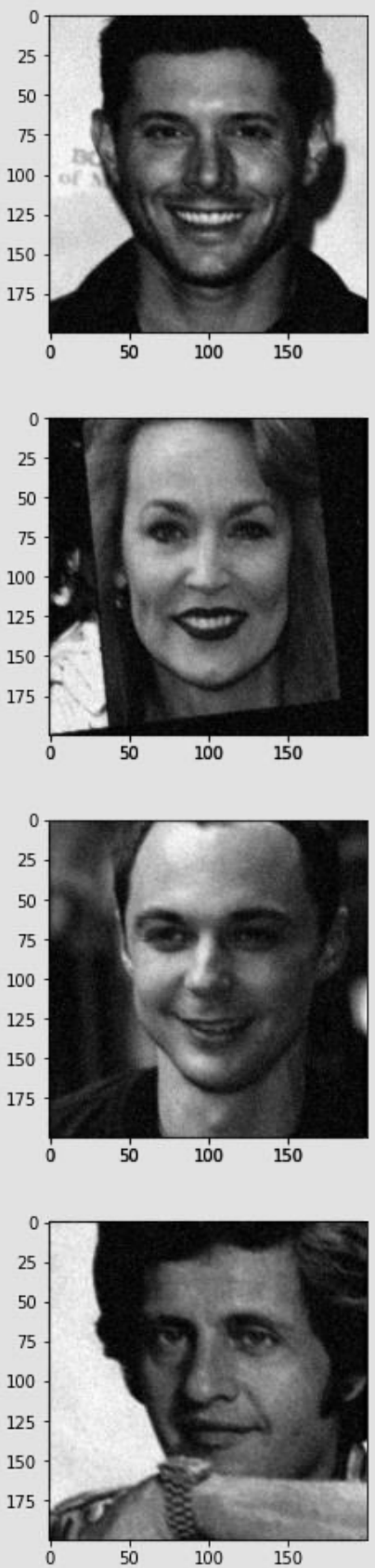

D
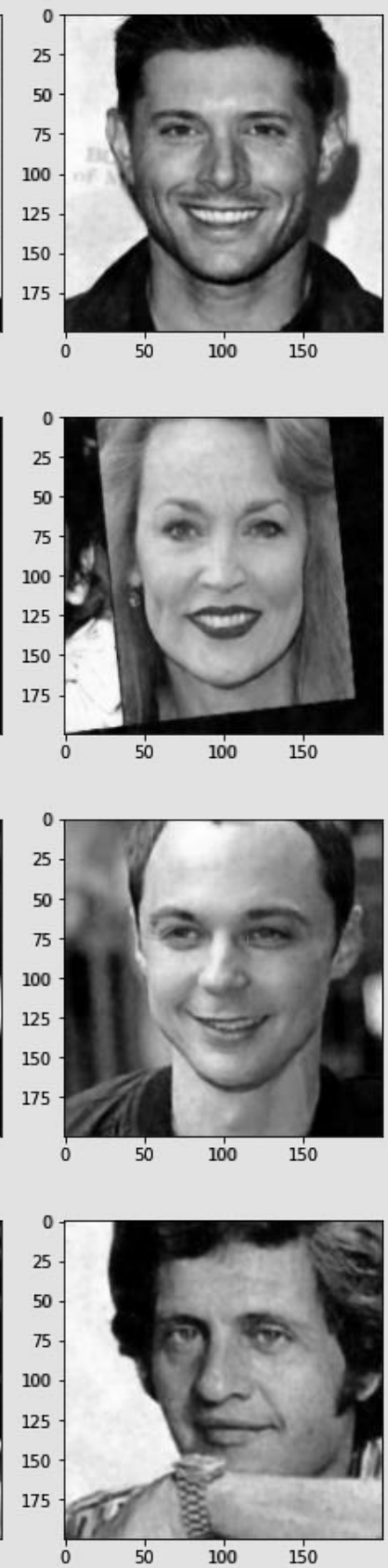

Рис. 6. А - исходное изображение; В - изображение восстановленное из голограммы к изображению А; C - изображение восстановленное из голограммы с добавлением шумового загрязнения; D результат восстановления искусственной нейронной сетью свёрточного автокодировщика изображения С. 
Ввиду конечности размеров реальной голограммы, восстановление изображения средствами машинного обучения так же носит приближенный характер. Кроме того при хранении и передаче голограммы на расстояние по каналу связи могут возникать искажения, которые желательно устранить. Критерием качества цифровой голографии является точность воспроизведение исходного изображения, т.е. величина квадрата нормы $\|U(x, y)-O(x, y, 0)\|^{2}$ в пространстве изображений. С учетом дискретизации данных виде пикселей, введем соответствие $U(x, y) \rightarrow U(n, m)$ и $O(x, y, 0) \rightarrow O(n, m)$. Тогда, считая изображения принадлежащими комплексному евклидовому пространству, примем оценку ошибки восстановления изображения в виде

$$
\begin{gathered}
\|U(n, m)-O(n, m)\|^{2}=\sum_{n, m}|U(n, m)-O(n, m)|^{2}= \\
=\sum_{n, m}|\operatorname{Re} U(n, m)-\operatorname{Re} O(n, m)|^{2}+\sum_{n, m}|\operatorname{Im} U(n, m)-\operatorname{Im} O(n, m)|^{2} .
\end{gathered}
$$

Поскольку финальное изображение на самом деле является комплексным и может содержать и другие искажения, то выбор того или иного фиксированного стандартного фильтра не гарантирует его оптимальность.

Формула (13) показывает, что оптимизация по действительной и мнимой части изображения может осуществляться раздельно. Поэтому, при использовании отдельной голографической записи действительной и мнимой составляющих, корректировку можно осуществлять целиком в поле действительных чисел. Если исправляется сама голограмма, то она описывается действительными числами и комплексность проявляется только на выходе в виде критерия качества (12).

\section{Список литературы}

1. D. Gabor. A new microscopic principle. Nature. 1948. V. 161. P. 777-778.

2. Poon, T., \& Liu, J. (2014). Introduction to Modern Digital Holography: With Matlab. Cambridge: Cambridge University Press. doi:10.1017/CBO9781139061346.

3. Ferdous Ahmed and Md. Sultan Mahmud. Fourier Optics and Computer Generated Hologram. International Journal of Computational Science and Engineering. 2249-4251 Volume 5, Number 1 (2015), pp. 1-7.

4. John G. Sutherland. International Journal of Neural Systems. Vol. 01, No. 03, pp. 259-267 (1990) A HOLOGRAPHIC MODEL OF MEMORY, LEARNING AND EXPRESSION. 
5. K.-Y. Hsu, H.-Y. Li and D. Psaltis, "Holographic implementation of a fully connected neural network," in Proceedings of the IEEE, vol. 78, no. 10, pp. 1637-1645, Oct. 1990, doi: 10.1109/5.58357.

6. Ryota Izumi, Satoshi Ikezawa, and Kentaro Iwami, "Metasurface holographic movie: a cinematographic approach," Opt. Express 28, 23761-23770 (2020).

7. Chenliang Chang, Kiseung Bang, Gordon Wetzstein, Byoungho Lee, and Liang Gao, "Toward the next-generation VR/AR optics: a review of holographic near-eye displays from a human-centric perspective," Optica 7, 1563-1578 (2020).

8. Zhiqin Huang, Daniel L. Marks, and David R. Smith, "Out-of-plane computer-generated multicolor waveguide holography," Optica 6, 119-124 (2019).

9. Zhiqin Huang, Daniel L. Marks, and David R. Smith, "Polarizationselective waveguide holography in the visible spectrum," Opt. Express 27, 3563135645 (2019).

10. Р.С. Гонсалес, Р.Е. Вудс. Цифровая обработка изображений. М.: Техносфера, 2012. $-1103 \mathrm{c}$.

11. Yoshua Bengio, Li Yao, Guillaume Alain, and Pascal Vincent. 2013. Generalized denoising auto-encoders as generative models. In Proceedings of the 26th International Conference on Neural Information Processing Systems Volume 1(NIPS'13). Curran Associates Inc., Red Hook, NY, USA, 899-907.

12. Solimeno S., Crosignani B., Di Porto P. Guiding, Diffraction and Confinement of Optical Radiation. - Orlando: Academic Press, 1986.—620 p.

13. Kim, M. (2011). Twin-image elimination using quadrantal masks in a digital holographic microscope. Optical Engineering, 50(2), 025801. doi:10.1117/1.3537970

14. Hennelly, B., Kelly, D., Pandey, N., \& Monaghan, D.S. (2009). Review of Twin Reduction and Twin Removal Techniques in Holography.

15. T. Latychevskaia and H. Fink, "Solution to the twin image problem in holography,” Phys. Rev. Lett. 98, p. 23390, 2007.

16. Yuxuan Zhang and Xinyi Zhang, "Reconstruction of a complex object from two in-line holograms," Opt. Express 11, 572-578 (2003)

17. Rong, $\mathrm{Lu}$, and $\mathrm{Da}$ Yong Wang. "Wavefront Reconstruction Using Two In-Line Holograms." Advanced Materials Research, vol. 718-720, Trans Tech Publications, Ltd., July 2013, pp. 1324-1328. Crossref, doi:10.4028/www.scientific.net/amr.718-720.1324.

18. Лейт Э, Упатниекс Ю "Фотографирование с помощью лазера" УФН 87 521-538 (1965). 
19. Rivenson, Y., Zhang, Y., Günaydın, H. et al. Phase recovery and holographic image reconstruction using deep learning in neural networks. Light Sci Appl 7, 17141 (2018). https://doi.org/10.1038/lsa.2017.141

20. Rivenson Y, Wu Y, Ozcan A. Deep learning in holography and coherent imaging. Light Sci Appl. 2019;8:85. Published 2019 Sep 11. doi:10.1038/s41377019-0196-0

21. Haoran Ren, Wei Shao, Yi Li, Flora Salim and Min Gu. Threedimensional vectorial holography based on machine learning inverse design. Science Advances 17 Apr 2020: Vol. 6, no. 16, eaaz4261 DOI: 10.1126/sciadv.aaz4261.

22. M. Hossein Eybposh, Nicholas W. Caira, Mathew Atisa, Praneeth Chakravarthula, and Nicolas C. Pégard, "DeepCGH: 3D computer-generated holography using deep learning," Opt. Express 28, 26636-26650 (2020).

23. Go, T., Lee, S., You, D. et al. Deep learning-based hologram generation using a white light source. Sci Rep 10, 8977 (2020). https://doi.org/10.1038/s41598020-65716-4.

24. Zhenbo Ren, Zhimin Xu, Edmund Y. M. Lam, "End-to-end deep learning framework for digital holographic reconstruction," Adv. Photon. 1(1) 016004 (28 January 2019) https://doi.org/10.1117/1.AP.1.1.016004.

25. Pavel Cheremkhin, Nikolay Evtikhiev, Vitaly Krasnov, Vladislav Rodin, Dmitry Rymov, Rostislav Starikov, Machine learning methods for digital holography and diffractive optics, Procedia Computer Science, Volume 169, 2020, Pages 440444.

(C) П.А. Головинский, А.С. Тарасова, 2022 